NASA Technical Memorandum 84241

NASA-TM-84241 19820017386

\title{
Barriers and Dispersal Surfaces in Minimum-Time Interception
}

N. Rajan and M. D. Ardema

May 1982

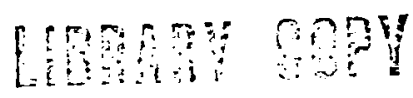

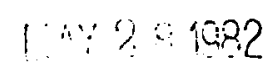

LANGLEY RESEARO $\because:$ R

LERERY, MASA

HrimeTon, V!ran:iA 
. 


\section{Barriers and Dispersal Surfaces in Minimum-Time Interception}

N. Rajan

M. D. Ardema, Ames Research Center, Moffett Field, California 


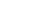


Barriers and Dispersal Surfaces in Minimum-Time Interception ${ }^{1}$

\title{
N. RAJAN ${ }^{2}$ AND M. D. ARDEMA ${ }^{3}$
}

\begin{abstract}
Dispersal points and points on the Barrier are located for a class of pursuit-evasion and interception problems. These points are determined by constructing cross sections of the isochrones and hence obtaining the Barrier, Dispersal, and controllevel surfaces. The method is illustrated through examples from minimum-time interception of a target moving in a straight line in a horizontal plane.
\end{abstract}

Key Words. Barriers, minimum-time interception, aircraft flight paths optimization.

\section{Introduction}

The area of optimizing aircraft maneuvers is quite complex and has received extensive attention. In Ref. 1, minimum-time turns to a line or a point in the plane are investigated when the final velocity and heading are specified. The destination point or line is taken far away from the starting point so that the aircraft has sufficient time to accelerate to maximum speed in all cases. The solution is obtained by integrating the optimal trajectory backward from termination. The assumption of long-duration makes the extremals

\footnotetext{
${ }^{1}$ The authors are indebted to F. Neuman, M. Sidar, J. Shinar, H. Erzberger, and G. Leitmann for their comments. The first author wishes to acknowledge the financial support of the National Research Council.

${ }^{2}$ NRC Research Associate, Ames Research Center, NASA, Moffett Field, California. ${ }^{3}$ Research Assistant to the Director, Ames Research Center, NASA, Moffett Field,
California.
} 
a one-parameter family. The two-boundary-value problem of choosing the extremal that satisfies the given initial conditions is thus simplified. Under the restriction of long-duration maneuvers, time-scale separation of turning and acceleration is possible, and singular perturbation approaches (Refs. 2, 3) have been successful in obtaining feedback approximations. However, shortand intermediate-duration maneuvers do not show up in these analyses. Aircraft maneuvers in a tall-chase pursuit-evasion game have been investigated in Ref. 4, employing a differential dynamic programming algorithm. The numerical: examples show tail-chase maneuvers of short duration.

In this paper, minimum-time interception of a target moving in a horizontal plane is analyzed as a one-player differential game (Ref. 5). The game solution gives the interceptor's strategy in a feedback form. It first addresses the question whether the target can be captured from all initial positions relative to the interceptor. If not, the Barrier, a closed-surface bounding the initial positions from which capture is possible, is constructed. Even if capture is possible for all initial states, a Barrier may exist, but in this case it is an open surface across which the game value, here the time-tocapture, is discontinuous, and the nature of the optimal trajectories changes drastically.

The game solution maps the controls as a function of the state within the capture region. This is done by backward integration of the state and adjoint equations from termination. The integration has to be stopped at surfaces where the adjoints are discontinuous; such surfaces are called singular surfaces (Ref. 5). One of the most frequently encountered singular surfaces is the Dispersal surface. Along such a surface, one player can choose between two distinct strategies and still obtain the same payoff. Constructing the Barrier and Dispersal surfaces is therefore an inherent part of a differential game solution. 
In a game with four or five state variables, visualization of the Barrier and Dispersal surfaces is difficult. This is circumvented by constructing the cross sections of these surfaces with two or more of the state variables held constant. A stack of such cross sections then describes the surfaces. This paper describes a method of computing the locations of points on these cross sections. Although minimum-time interception in the plane is used as an example, the extension of the method to pursuit-evasion in the plane, which is straightforward, is also outlined.

The analysis uses a device first developed in Refs. 6-8. The motion of the interceptor and the target are described in a coordinate system whose origin is fixed at the interceptor's terminal position, and the $x$-axis is along the terminal line of sight. The equations that describe the interceptor's optimal motion backward in time from termination do not contain any reference to the target motion. They can be integrated independently to give extremal trajectory maps (ETM) (Ref.6). Using this concept, cross sections of the Barrier were constructed by direct iteration of the terminal Mach numbers of the pursuer and evader in Refs. 6-8. In this paper, constant initial Mach number and relative heading cross sections of the isochrones (constant-time loci) are drawn. At Dispersal points (DP), two branches of an isochrone intersect. An isochrone ends if capture becomes impossible for the given timeto-go. The endpoints thus fall on a Barrier cross section.

The interception problem is formulated and the necessary conditions for optimality are derived in Section 2. Extremal trajectory maps are shown for a given inftial interceptor speed. The method of constructing a global solution is described in Section 3. Isochrone cross sections for interception are depicted, and some interception encounters in the plane are shown. The extension of the method to pursuit-evasion is outlined. 


\section{Problem Statement}

2.1 Formulation. The motion of the target in the plane is described in terms of its coordinates $\left[x_{T}(t), y_{T}(t)\right]$ in any conventient set of axes. Since the motion is prescribed a priori, the target motion is a function of time alone. In the same coordinate frame, the interceptor's motion is goverened by

$$
\begin{gathered}
\dot{x}_{I}=M_{I} \cos \beta_{I}, \\
\dot{y}_{I}=M_{I} \sin \beta_{I}, \\
\dot{M}_{I}=A\left(M_{I}\right) \pi-B\left(M_{I}\right)-C\left(M_{I}\right) \omega^{2}
\end{gathered}
$$

and

$$
\dot{\beta}_{I}=\omega a\left(M_{I}\right) / M_{I}
$$

subject to the state-variable constraint,

$$
M_{I} \varepsilon[\underline{M}, \bar{M}]
$$

with the throttle setting $\pi$ and the bank control $\omega$ chosen such that

$$
\pi \varepsilon[0,1] \text { and } \omega \varepsilon[-1,1]
$$

The above description of the aircraft's motion is the same as that given in Ref. 6. Briefly, $\beta_{I}$ is the interceptor's heading measured from the $x$-axis (counterclockwise positive); $M_{I}$ is the Mach number; A represents the maximum thrust, $B$ is the zero bank drag, $C$ is the lift-induced drag at the maximum bank, and $a$ is the angular acceleration. The target is considered to have been intercepted when brought within a line of sight (LOS) distance of $R$ of the interceptor. This is, 


$$
\left[x_{T}\left(t_{f}\right)-x_{I}\left(t_{f}\right)\right]^{2}+\left[y_{T}\left(t_{f}\right)-y_{I}\left(t_{f}\right)\right]^{2}=R^{2}
$$

at the instant of interception $t_{f}$, the interceptor must be reducing the separation distance or

$$
\dot{r}=M_{T}\left(t_{f}\right) \cos \beta_{T}\left(t_{f}\right)-M_{I}\left(t_{f}\right) \cos \beta_{I}\left(t_{f}\right)<0
$$

to effect capture. Where interception occurs, the payoff is the time taken to capture the target. The performance index, augmented by the terminal constraint (6), is

$$
J_{a}=v\left[\left(x_{T}\left(t_{f}\right)-x_{I}\left(t_{f}\right)\right)^{2}+\left(y_{T}\left(t_{f}\right)-y_{I}\left(t_{f}\right)\right)^{2}-R^{2}\right]+\int_{o}^{t} f_{o} d t .
$$

As in the analyses of pursuit-evasion, (Refs. 6-8) the coordinate axes, to which the above equations refer, are selected such that the origin coincides with the interceptor's terminal position, and the $x$-axis is along the terminal line of sight (Fig. 1). The position and orientation of the axes thus change from engagement to engagement. As is shown below, this choice of axes makes the trajectory equations and the expressions for the optimal controls for the interceptor independent of the target motion. The analysis is simplified and leads very naturally to the method of locating Dispersal points, which is developed later.

2.2 The Necessary Conditions. The Hamiltonian for the minimum-time interception problem is written as

$$
H=1+\lambda_{x} \dot{x}_{I}+\lambda_{y} \dot{y}_{I}+\left(\lambda_{M}+\mu\right) \dot{M}_{I}+\lambda_{B} \dot{B}_{I},
$$

where $\lambda_{x}, \lambda_{y}$, are the position adjoints; $\lambda_{M}$ and $\lambda_{B}$ are the velocity and heading adjoints; and the multiplier $\mu$ accounts for the first-order statevariable (Ref. 9) constraint on the interceptor's Mach number. It satisfies the Kuhn-Tucker conditions, 


$$
\begin{aligned}
<0 & \text { if } \underline{M}_{I}=\underline{M}, \\
\mu \quad & 0 \text { if } \underline{M}<M_{I}<\bar{M}, \\
>0 & \text { if } M_{I}=\bar{M} .
\end{aligned}
$$

From the Euler-Lagrange equations and the transversality conditions plus the particular choice of coordinate axes mentioned above, it can be shown that the adjoints satisfy (Appendix)

$$
\begin{gathered}
\lambda_{x}=-2 \cup R, \\
\lambda_{y} \equiv 0,
\end{gathered}
$$

and

$$
\lambda_{B}=-2 v \mathrm{Ry}_{\mathrm{I}} \text {. }
$$

The vanishing of the Hamiltonian at $t_{f}$ gives the value of the multiplier $v$ as

$$
v=\left(2 R\left(M_{I f} \cos \beta_{I f}-M_{T f} \cos \beta_{T f}\right)\right)>-j
$$

$V$ is positive because the target's velocity relative to the interceptor has a negative component along the line of sight at the endpoint.

Because $H$ does not involve time explicitly, a first integral of motion is given by (Appendix)

$$
\underset{(\pi, \omega)}{\min H}=-2 \nu \mathrm{R}_{\mathrm{Tf}} \cos \beta_{\mathrm{Tf}}
$$

When the normalized velocity adjoint $\rho_{M}$ and the multiplier $\bar{\mu}$ are defined, repectively, as

$$
\rho_{M} \stackrel{\Delta}{=} \lambda_{M} / 2 \nu R
$$


and

$$
\bar{\mu} \triangleq \mu / 2 v R \text {, }
$$

and by using (11)-(13) in (9), then (15) becomes

$$
\min _{(\pi, \omega)} H_{I}^{\prime}=-M_{I f} \cos \beta_{I f},
$$

where

$$
H_{I} \triangleq\left(\rho_{M}+\bar{\mu}\right) \dot{M}_{I}-\dot{x}_{I}-y_{I} \dot{\beta}_{I}
$$

Minimizing $H_{I}$ with respect to $(\pi, \omega)$ subject to the constraints $\pi \varepsilon[0,1]$, $\omega \in[-1,1]$ yields the following expression for the optimal controls:

$$
\begin{gathered}
\pi=1 \\
\omega=\operatorname{sat}\left(-\operatorname{ay}_{I} /\left(2 \mathrm{CM}_{I}\left(\rho_{M}+\bar{\mu}\right)\right)\right) \text { for } \rho_{M}+\bar{\mu}<0
\end{gathered}
$$

and

$$
\begin{gathered}
\pi=0 \\
\omega=\operatorname{sign}\left(y_{I}\right) \text { for } \rho_{M}+\bar{\mu}>0 .
\end{gathered}
$$

The normalized velocity adjoint appearing in the above expressions satisfies the differential equation

$$
\begin{gathered}
\dot{\rho}_{M}=\cos \beta_{I}-\left(\rho_{M}+\bar{\mu}\right)\left(-d A / d M_{I}-\omega^{2} d c / d M_{I}\right)+ \\
\omega y_{I}\left(d a / d M_{I}-a / M_{I}\right) / M_{I}
\end{gathered}
$$

Note that the canonical equations (1), $-(4)$, and (22), together with the controls (20) and (21) contain no reference to the target motion; the same type of "open-1oop optimality" found in pursuit-evasion is obtained here. 
Backward integration of the extremals depends only on the terminal-velocity vector of the interceptor and is independent of target motion. Thus a map of the extremals may be generated integrating backward from the origin; this is called the extremal trajectory map (ETM) in Refs. 6 and 7.

2.3 Extremal Trajectory Maps. Each extremal in an ETM may be considered to be specified by a triplet of values $\left(\beta_{f}, M_{f}, \tau_{f}\right)$, where $\beta_{f}$ and $M_{f}$ are the terminal heading and Mach number, and $\tau_{f}\left(=t_{f}-f\right)$ is the time-to-go. For any given pair of values for $\beta_{f}$ and $\tau_{f}$, it is possible to select an $M_{f}$ value, through a one-dimensional search, such that the Mach number at $\tau_{f}$ matches a given value $M_{0}$. The ETMs used here are collections of extremals that start out from a given initial Mach number. They can be combined with a target/pursuer/evader's ETM to yield a global solution for any given encounter. For this, apart from the triplet $\left(\beta_{f}, M_{f}, \tau_{f}\right)$, the initial coordinates $(x, y)$ and heading $\beta$ are needed. The behavior of the bank and throttle along the extremals determines the values of $\tau_{f}$ for which isochrones need be drawn (Section 3).

The trajectories in the plane for $\tau_{f}=10 \mathrm{sec}$ are shown in Fig. 2a. The variation of Mach number, heading and bank with retro time is shown in Figs. $2 b-2 d$. For $\beta_{f}=0^{\circ}$, the aircraft flies in a straight line at full throttle, accelerating from Mach 0.9 to Mach 1.02. The extremal with $\beta_{f}=0.33^{\circ}$ starts out at half-bank and gradually straightens out to nearlevel flight. The angle of turn $\Delta \beta=23^{\circ}$, and the terminal Mach number is slightly above unity. On the next extremal, the bank decreases from unit magnitude at start to below 0.1 at termination; $\Delta B=45^{\circ}$, and the average turn rate is about half the maximum instantaneous at Mach 0.9 . As $B_{f}$ increases beyond $0.85^{\circ}$, the bank saturation instant moves closer to termination, till at $\beta_{f}=15^{\circ}$, the entire trajectory is flown at full bank. 
These trajectories represent different trade-offs between turn and speed. However, their initial points would not appear on the cross section of the unit bank control surface taken with the initial Mach number equal to 0.9. Between $\beta_{f}=15^{\circ}$ and $77^{\circ}$, the extremals are constant control arcs; both bank and throttle are full throughout. The Mach number time-history is identical for all extremals in this range of $\beta_{f}$ and is hence a single curve in Fig. 2b. The turn angle $\Delta \beta=109^{\circ}$, or the average turn rate $=10.9^{\circ} / \mathrm{sec}$. Between $77^{\circ}$ and $90^{\circ}$, the extremals start out with the throttle closed and switch to full throttle sometime later; this switching instant moves toward $t_{f}$ with increasing $\beta_{f}$ till, at $90^{\circ}$, the extremals terminate with zero throttle. The region between $\beta_{f}=90^{\circ}$ and $100^{\circ}$, shows throttle switches from zero to full and back to zero; for $\beta_{f}=100^{\circ}$, at $\tau=9.5(0$ to 1$)$ and $6.6(1$ to 0$) \mathrm{sec}$, respectively. Beyond $\beta_{f}=100^{\circ}$, the extremals are all flown at zero throttle, full bank; the Mach number variation is independent of $\beta_{f}$ (Fig. 2b).

The $140^{\circ}$ and $160^{\circ}$ extremals show switchings in bank (Fig. 2c) where they intersect the $x$-axis. Such maneuvers reduce the turn angle; the net effect is to brake down while heading in the same direction. Such a maneuver is resorted to when the target is close behind and heading in the same direction. In Fig. 2a, the extremals for $\beta_{f}=140^{\circ}$ and $160^{\circ}$ appear to intersect. However, the heading angles at the point of intersection are different, as can be seen from Fig. 2c.

Bank and throttle switching disappear as $\tau_{f}$ increases beyond $25 \mathrm{sec}$. The behavior of the extremals with increasing $\tau_{f}$ becomes more uniform and can be summarized as follows: as $\tau_{f}$ increases, the values of $\beta_{F}$ for the same $\Delta \beta$ decrease steadily (for $\tau_{f}=60 \mathrm{sec}, \beta_{f}=0.0005^{\circ}$ for $\Delta \beta=200^{\circ}$ ); all extremals have partial bank segments and are full-throttle throughout for the 
initial Mach number of 0.9. As $\tau_{f}$ increases further, the terminal Mach number hits the speed boundary. The extremals then consist of an unconstrained (maximum Mach number constraint) arc accelerating up to $\bar{M}$, a circular arc at $\bar{M}$, then a straight dash at $\bar{M}$ along the negative $x$-axis. Such extremals, called cruise arcs, are similar to those discussed in Ref. 1. However, the use of these extremals to locate Dispersal points depends only on a decoupling coordinate transformation approach being possible; it is discussed next.

\section{Construction of a Feedback Solution}

A feedback solution to interception or pursuit-evasion must give the control strategies and the value at any point in the state-space. One way of presenting the solution is to draw the boundaries of the regions within which the strategies are constant. Where a control varies continuously over a range, as does the bank here, the surfaces at which the control attains specified values are mapped. Since these boundaries and surfaces are in a four or five dimensional state-space, they can be visualized by cross sectioning. They are sectioned by keeping the initial Mach numbers of the interceptor and target, or of the pursuer and evader, and the initial relative heading, constant. The cross sections are then plotted relative to the interceptor/pursuer and become curves in the plane of the encounter.

The control-level surfaces, Barrier Points (BP), and Dispersal Points are all located by drawing cross sections of the isochrones (the optimal parts of the isochrones are isocost). The control-level surface links the points on the isochrone at which the controls take on a specified set of values. Dispersal Points are located at the intersections of different branches of the same isochrone. Where the isochrone cross sections are not closed, their 
endpoints are on a Barrier cross section. Such a section separates two families of isochrones. The construction of the isochrone sections for interception is described next.

3.1 Isochrones for Interception. Assume that the interceptor and target ETMS- are as shown in F1gs. $3 a$ and $3 b$, respectively. The interceptor's map comprises extremals generated for a specified time to go with the terminal heading $B_{f}$ as a parameter. For each extremal in the map, the initial Mach number is $\mathrm{M}_{\text {Io }}$; the initial heading measured relative to the terminal line of sight equals $\beta_{\text {Io }}$. The target's initial Mach number is given as $M_{\text {To }}$. Since the target's motion is prescribed, the target's terminal Mach number M and the angle of turn $\Delta R_{T}$ at $\tau_{f}$ are known. Although the target's motion in the plane is fixed, its heading relative to the terminal line of sight $\beta_{T f}$ varies with the different paths flown by the interceptor. The target's ETM consists of the target's path in the plane rotated through several different values of $\beta_{\text {Tf }}$ (the term ETM is used only as a convenience).

If the relative heading of the isochrone section being constructed is $\beta_{0}$, then the initial heading of the target measured from the terminal Los must be equal to $B_{I 0}+B_{0}$. Since the target's angle of turn is $\Delta B_{T}$, its terminal heading equals $\beta_{\text {Io }}+\beta_{0}-\Delta \beta_{T}$. In the construction of a locus point (Fig. $3 c$ ), the interceptor's extremal is first laid off as IA. The terminal LOS is AB. Since the target's heading relative to $A B$ is known, it can be laid off as shown, TB. The point $T$ is the point on the locus, provided the terminal Los rate is negative; that is

$$
M_{I f} \cos \left(\beta_{I 0}+\beta_{o}-\Delta \beta_{T}\right)-M_{I f} \cos \beta_{f}<0
$$

Different values of $\beta_{f}$ give other points on the locus. Points of interest on the extremals, for example bank saturation, throttle, and bank 
switching, also get mapped on the isochrone locus section. For a section with a different relative heading, these points are rotated by the difference in $\beta_{0}$. The method plots all candidate points for the isochrone. This is true because extremals for all possible values of $\beta_{f}$ are generated. Some of the resulting points are not optimal because there are other constant-time loci points for a lower time-to-go overlapping them. A Dispersal point occurs if two extremals with different values of $\beta_{f}$ pass through the same point in reduced space. Neither extremal is globally optimal beyond the DP.

The location of Dispersal and Barrier points and the construction of isochrones are explained by examples.

3.2 Examples. Figure $4 a$ shows some isochrone section for an interceptor initial Mach number of 0.9 and a relative heading of $135^{\circ}$. The points. $A_{1}$ to $A_{4}$ are those for which $B_{F}=0^{\circ}$; the interceptor just flies straight ahead at full throttle to meet the target. To the right of these points, the interceptor banks to the right, and the terminal heading $B_{f}$ is positive. For all the sections, $B_{E}$ increases in the direction $C$ to $B$. The sections do not extend beyond the points B and C because the "reachability" condition is not met; thus, the envelope of these points delineates the $\beta_{0}=135^{\circ}, M_{\text {Io }}=0.9$ section of the Barrier (Ref. 5). However, since the interceptor has a maximum Mach number capability of 1.6 , it can capture the target, given sufficient time. This can be seen from Fig $4 b$ where the 30 sec locus is also shown. Across $B_{3}$, there is a discontinuity in the time-to-go. From $B_{3}$ to $P$, the time-to-go is $30 \mathrm{sec}$. The $P Q D_{1}$ portion of the constanttime locus for $t_{f}=30 \mathrm{sec}$ is nonoptimal and does not form part of any isochrone. All points on $P Q D_{1}$ are conjugate points; however, only $D_{1}$ and $P$ actually appear on the isochrone sections. This suggests that not all values of $\beta_{f}$ lead to extremals that are globally optimal for a given value of $\beta_{0}$; 
there are points for $\tau_{f}=20 \mathrm{sec}$ which fall between the origin and the 15sec locus and are hence obviously non-optimal globally.

Figure $4 c$ shows a more complete picture of the isochrone sections with $\tau_{f}$ ranging from 5 to $60 \mathrm{sec}$. The $60-\mathrm{sec}$ locus is the first one that is closed. Even for an initial range of $2 \mathrm{~km}, 60 \mathrm{sec}$ is the globally optimal time-to-capture because of the unfavorable initial conditions. The target has a higher initial speed and its direction is away from the interceptor. The interceptor turns left and accelerates to Mach 1.2 with its terminal heading almost aligned with the final LOS. This encounter starting from $P$ is depicted in real space in Fig. 5. The locus shows a Dispersal point at D. Extremals with two different values of $\beta_{f}$ pass through $D$; they are shown in the plane in Fig. 6 .

In Fig. 7, an isochrone section with $\beta_{o}=0^{\circ}$ and $\tau_{f}=20 \mathrm{sec}$ is shown. The Dispersal point at $D_{1}$ is one along a Dispersal surface where the interceptor flies symmetric paths starting with either a left or a right turn; the values of $\beta_{f}$ at $D_{1}$ are $\pm 116^{\circ}$. The encounter at $D_{1}$ is shown in Fig. 8 . Another interesting kind of Dispersal point occurs at $D_{2}$ in Fig. 7. Two branches of the locus, one with $\beta_{f}$ decreasing toward $90^{\circ}$ and the other with $\beta_{F}$ increasing, intersect at $D_{2}$. The encounter at $D_{2}$ is shown in Fig. 9 .

The additional computational effort to map the feedback solution, given the ETMs, is relatively small. Moreover the logic of determining the Barrier and Dispersal Points can be automated. The generation of the ETM data storage is a straightforward, though tedious process; once done, however, the data are common to any interception/pursult evasion problem in which the given aircraft is a participant. The method of constructing maps of strategies and isochrones can serve as a useful tool for design, displays, training, and testing of suboptimal approximations. The extension of the method to interception and 
pursuit-evasion in three dimensions is, in principle, stralghtforward. If the aircraft is modeled based on the energy-state approximation, with the specific energy replacing the Mach number as a variable, a similar structure is obtained. The extension of the method to pursult-evasion is outlined.

\subsection{Pursuit-Evasion. The above method of constructing isochrone} sections can be employed for an actively evading target. The cross sectioning would be done for fixed initial Mach numbers and relative heading for the pursuer and evader. The evader's ETM would be constructed in exactly the same way as the pursuer's, with $B_{f}, \tau_{f}$, as parameters and $M_{f}$ iterated till the initial Mach number is matched. For each point on a constant-time locus, the evader's ETM would be searched for the extremal that has an initial heading equal to $\beta_{0}+\beta_{10}$. The "reachability" condition would be checked for the extremal. This search for the extremal in the evader's ETM is independent of the search for the $M_{f}$ value that generates it. Other features of the method are the same as for interception.

\section{Conclusions}

A method of locating Barrier and Dispersal points for a class of minimumtime interception and pursuit-evasion problems has been developed. The method obtains these points, which are an essential part of the game solution, by drawing cross sections of the isochrones. Examples are shown for minimum-time interception in a horizontal plane. The method can be extended to solve pursuitevasion between two aircraft in the plane and in three dimensions. 
Appendix

The differential equations for the adjoints are

$$
\begin{gathered}
\dot{\lambda}_{x}=0, \\
\dot{\lambda}_{y}=0, \\
\dot{\lambda}_{M}=-\lambda_{x} \cos \beta_{I}-\lambda_{y} \sin \beta_{I}-\left(\lambda_{M}+\mu\right)\left(\pi d A / d M-d B / d M-\omega^{2} d C / d M\right) \\
+\dot{\lambda}_{B} \omega(a / M-d a / d M) / M,
\end{gathered}
$$

and

$$
\dot{\lambda}_{B}=\lambda_{x} \dot{y}_{I}-\lambda_{y} \dot{x}_{I}
$$

For the choice of coordinate axes shown in Fig. 1, the transversality conditions give

$$
\begin{gathered}
\lambda_{x}\left(t_{f}\right)=-2 \cup R, \\
\lambda_{y}\left(t_{f}\right)=\lambda_{B}\left(t_{f}\right)=\lambda_{M}\left(t_{f}\right)=0,
\end{gathered}
$$

and

$$
H\left(t_{f}\right)=-2 v M_{T f} \cos \beta_{T f} \cdot
$$

In (A7), $M_{T f}$ and $\beta_{\text {Tf }}$ are the target's terminal Mach number and heading. Equations (A1), (A2), and (A4), together with the end conditions (A5) and (A6), yield (11) - (13). The substitution of (A5) in (A7) leads to (14). 


\section{References}

1. BRYSON, Jr., A. E., and PARSONS, M. G. Constant Altitude Minimum-Time Turns to a line and to a Point for a Supersonic Aircraft with a on Maximum Velocity, SUDAAR No. 437, Stanford University, Nov. 1971.

2. CALISE, A. J., Singular Perturbation Techniques for On-Iine Optimal Flight Path Control, Paper 79-1621, AIAA Atmospheric Flight Mechanics Conference, Boulder, Colo., Aug. 6-8, 1979.

3. FARBER, N., and SHINAR, J., An Approximate Feedback Solution of a Variable Speed Non-Linear Pursuit-Evasion Game between Two Airplanes in a Horizontal Plane, Paper 80-1597, AIAA Atmospheric Flight Mechanics Conference, Danvers, Mass., Aug. 11-13, 1980.

4. JARMARK, B. S. A., MERZ, A. W. and BREAKWELL, J. V., The Variable-Speed Tail-Chase Aerial Combat Problem, AIAA Journal of Guidance and Control, Vol. 4, No. 3, May-June 1981, pp. 323-328.

5. ISAACS, R., Differential Games, John Wiley \& Sons, New York, 1965.

6. RAJAN, N., PRASAD, U. R., and RAO, N. J., Pursuit-Evasion of Two Aircraft in a Horizontal Plane, AIAA Journal of Guidance and Control, Vol. 3, No. 3, May-June 1980, pp. 261-267.

7. PRASAD, U. R., RAJAN, N. and RAO, N. J., Planar Pursuit-Evasion with Variable Speeds, Part 1, Extremal Trajectory Maps, Journal of Optimization Theory and Applications, Vol. 33, No. 3, Mar. 1981, pp. 401-418.

8. RAJAN, N., PRASAD, U. R., and RAO, N. J., Planar Pursuit-Evasion with Variable Speeds, Part 2, Barrier Sections, Journal of Optimization Theory and Applications, Vol. 33, No. 3, Mar. 1981, pp. 419-432.

9. BRYSON, Jr., A. E., and HO, Y. C., Applied Optimal Control, John Wiley, 1975 (revised). 
List of Figure Captions

Fig. 1. Choice of corrdinate axes.

Fig. 2. Extremal trajectory map: (a) paths in the plane; (b) Mach number time history; (c) heading time-history; (d) bank control timehistory.

Fig. 3. Construction of a point on a constant-time 1ocus.

Fig. 4. Constant-time loci in reduced space: (a) loci for 5, 10, 15, and 20 secs; (b) additional 30-sec locus with nonoptimal segments;

(c) Barrier and Dispersal points.

Fig. 5. Long-duration encounter starting close-in.

Fig. 6 Interceptor paths for a DP on the 1-min locus.

Fig. 7. A $20 \mathrm{sec}$ locus with 2 DPs.

Fig. 8. Interceptor path with bank switching.

Fig. 9. The two possible paths from the Dispersal Point $D_{2}$. 


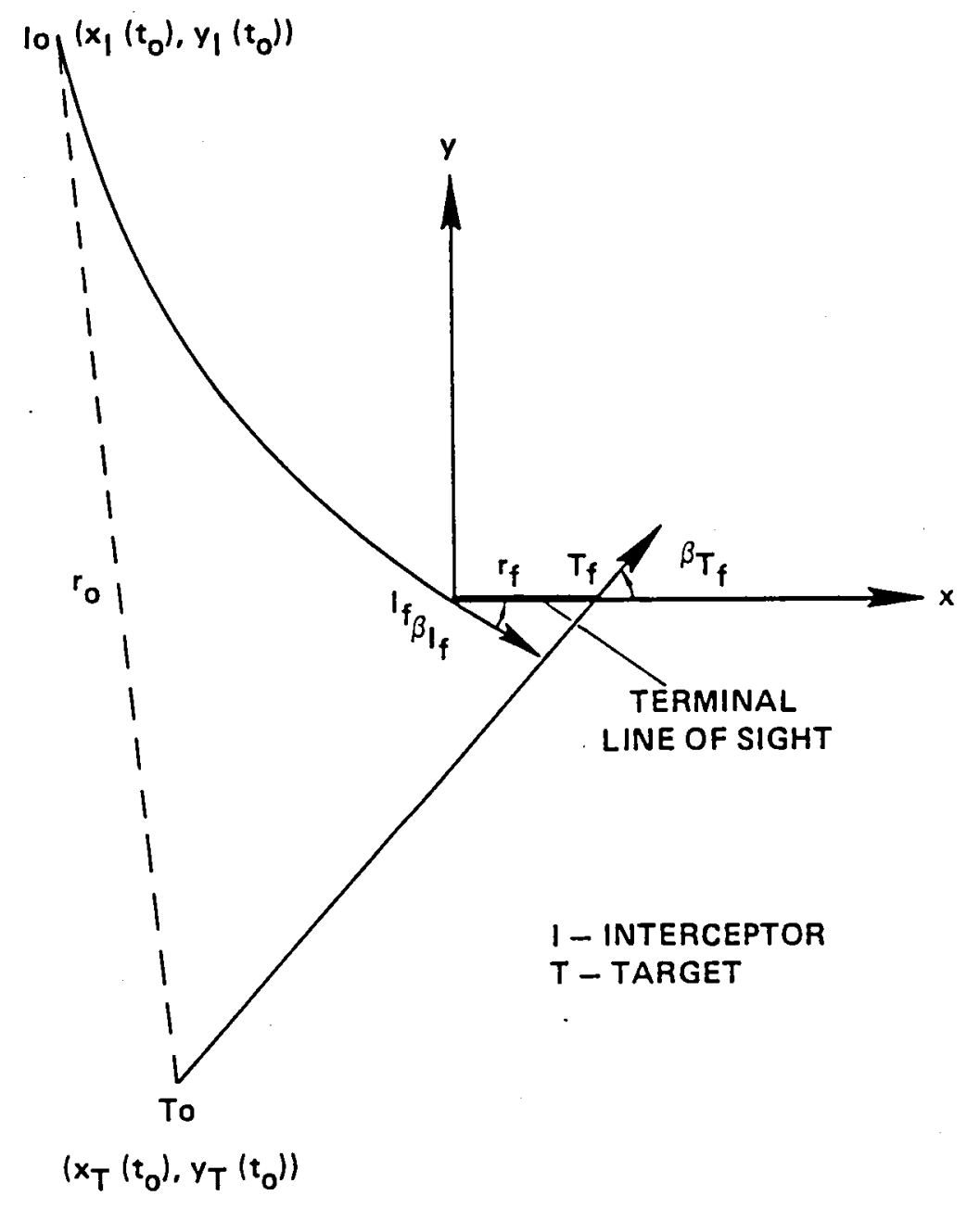

Fig. 1 


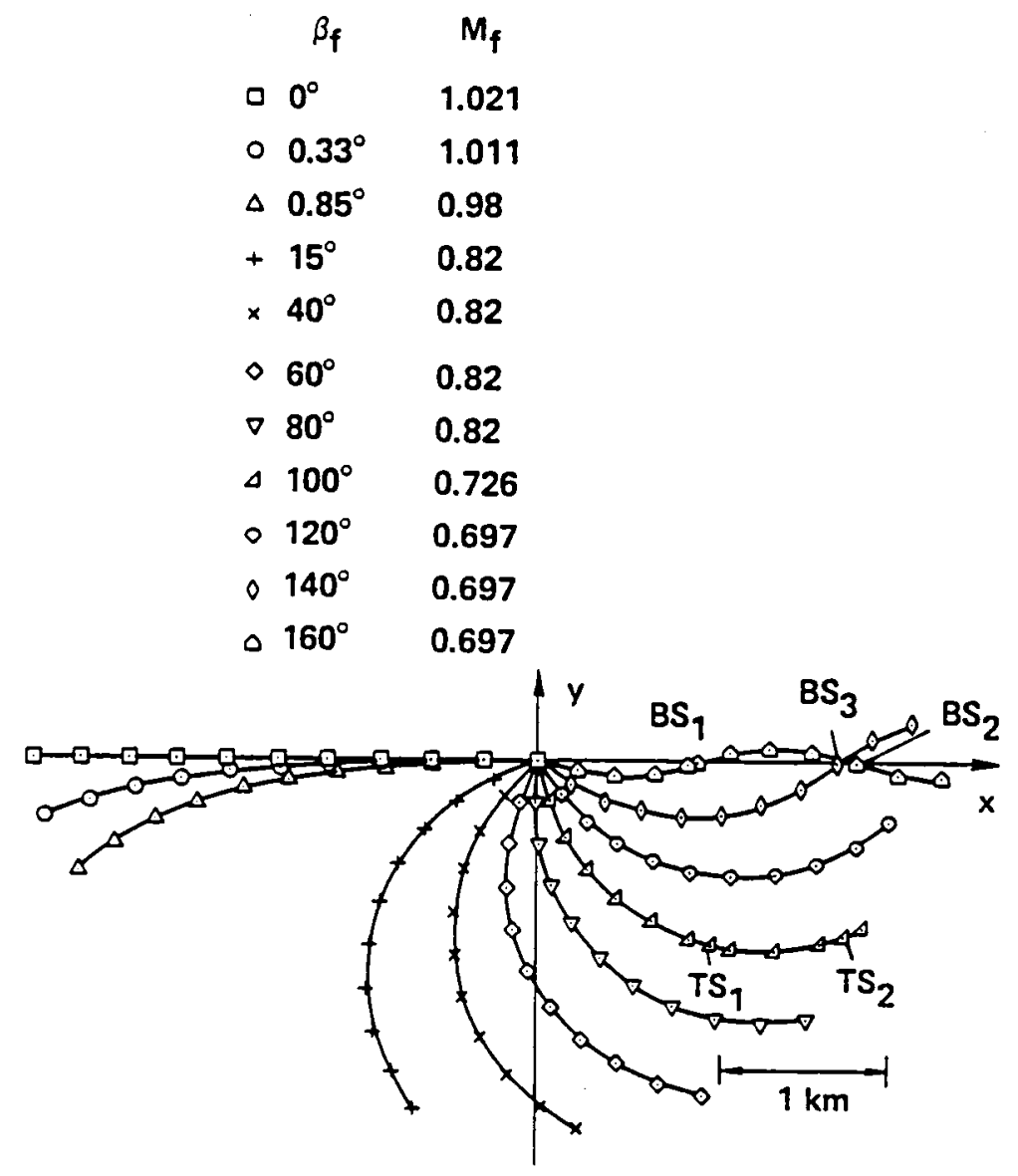

$\mathrm{BS}_{1}, \mathrm{BS}_{2}, \mathrm{BS}_{3}-$ BANK SWITCH POINTS

$\mathrm{TS}_{1}, \mathrm{TS}_{2}-\mathrm{THROTTLE} \mathrm{SWITCH} \mathrm{POINTS}$

Fig. 2a 


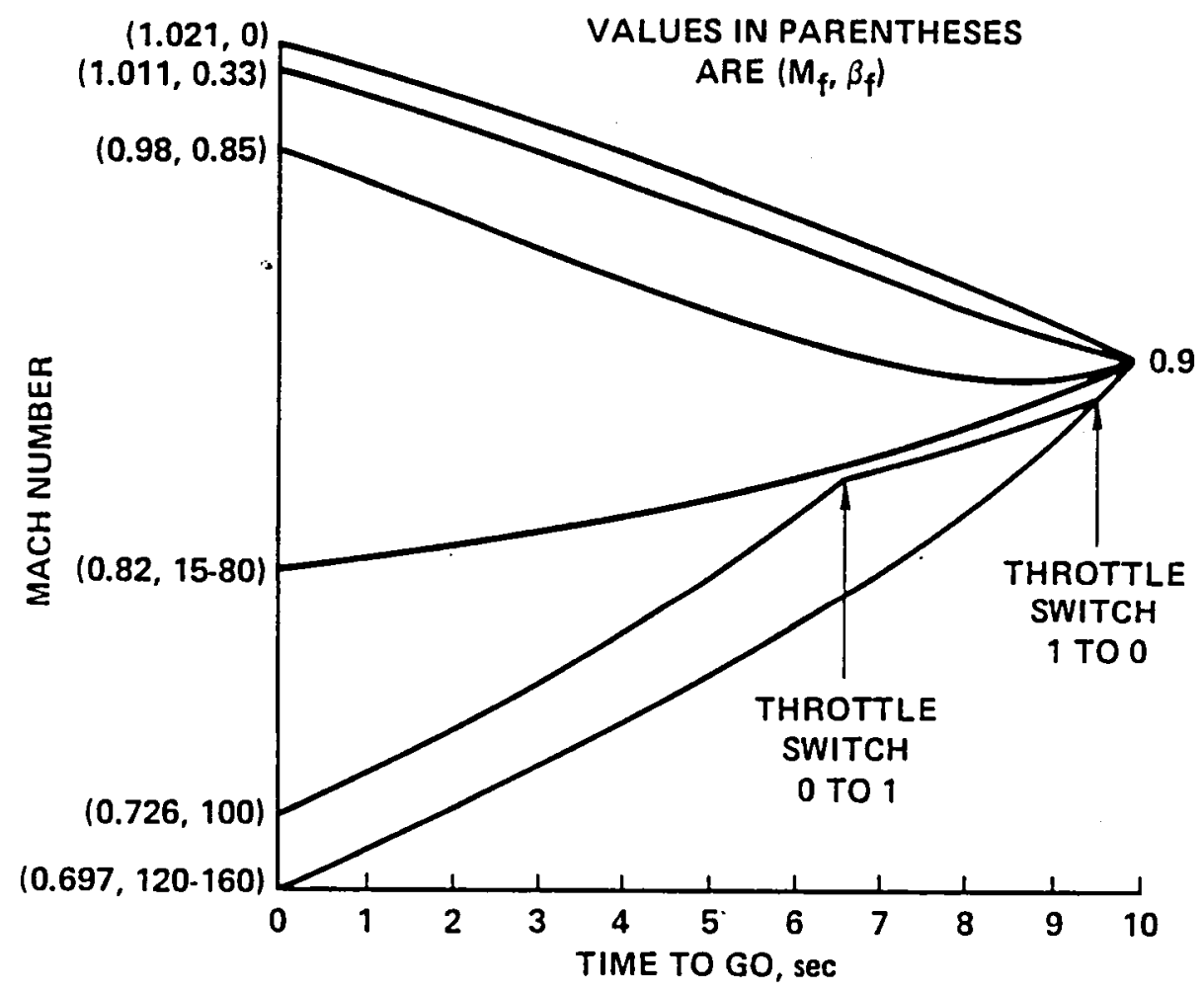

Fig. 2b 


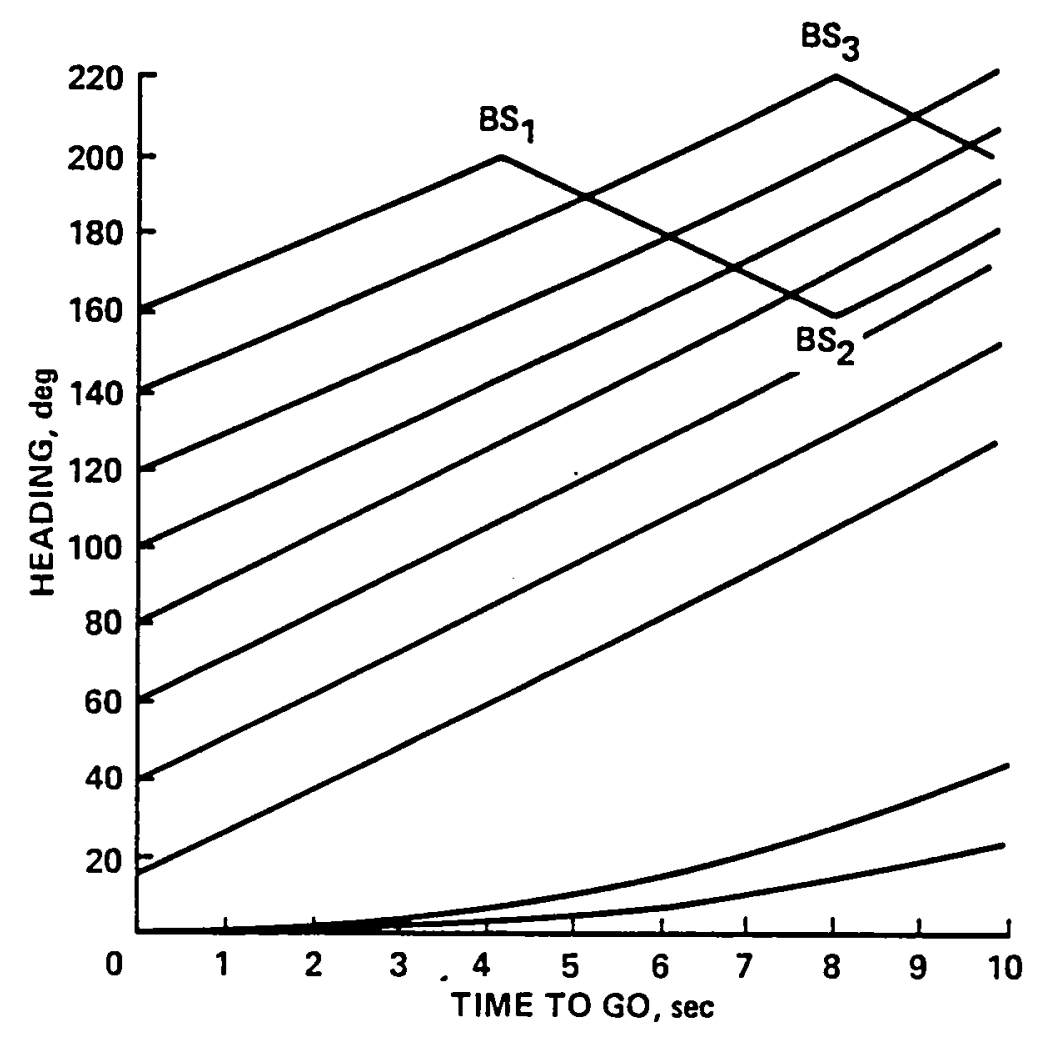

$\mathrm{BS}_{1}, \mathrm{BS}_{2}, \mathrm{BS}_{3}-\mathrm{BANK}$ SWITCH POINTS

Fig. 2c 


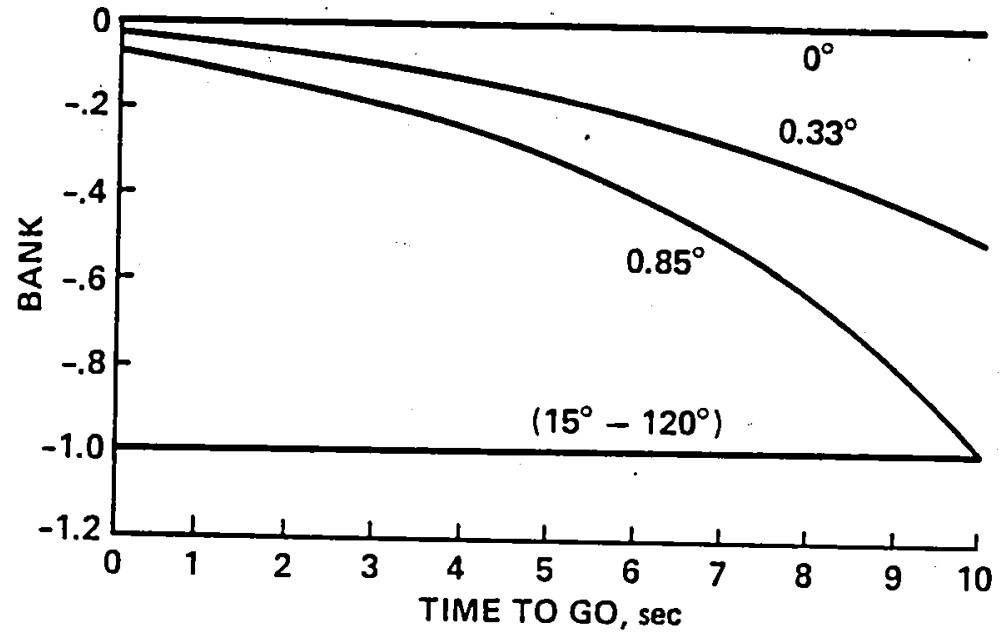

( $\beta_{f}$ MARKED ALONG THE CURVE)

Fig. 2d 


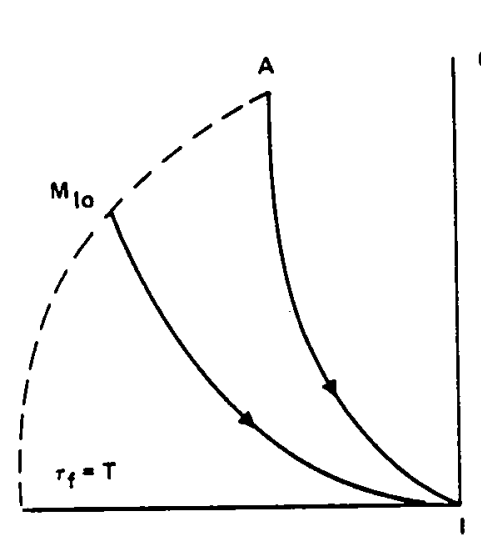

(a) INTERCEPTOR'S ETM

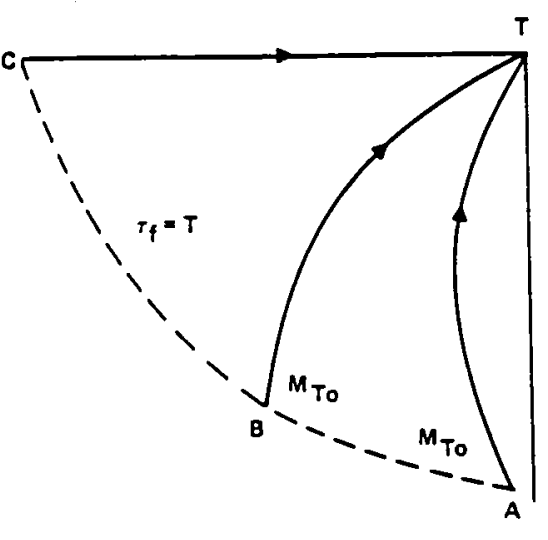

(b) TARGET'S ETM

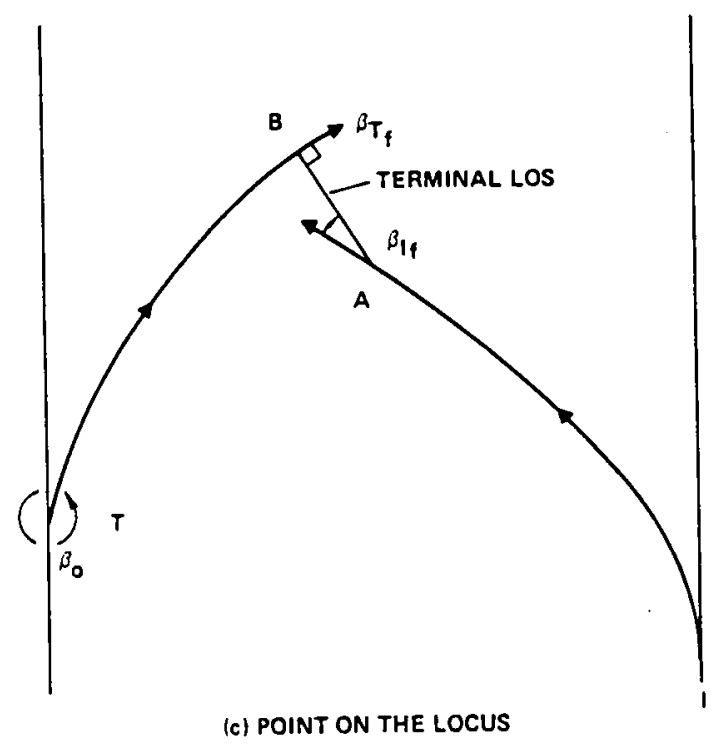

Fig. 3 


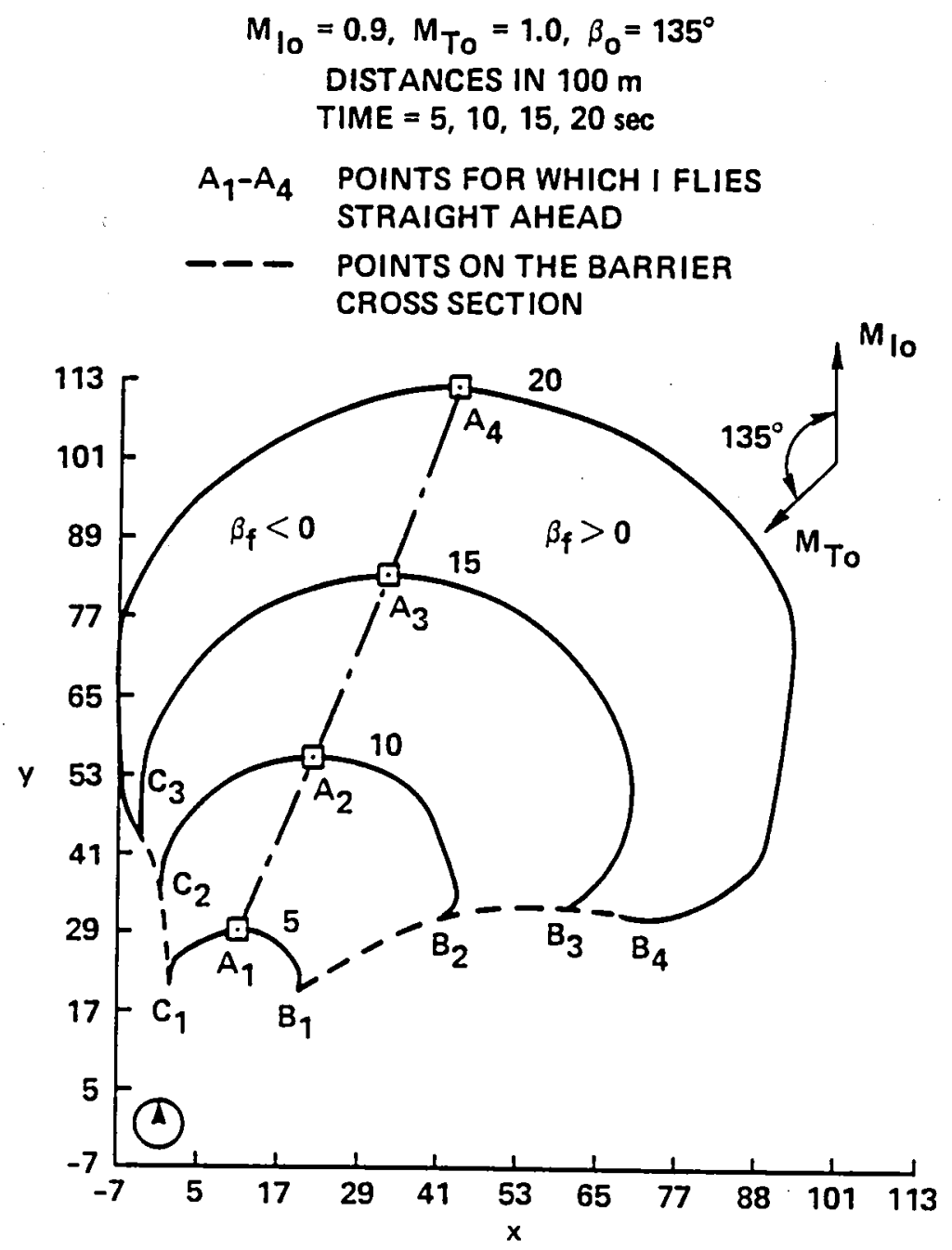

Fig. $4 a$ 


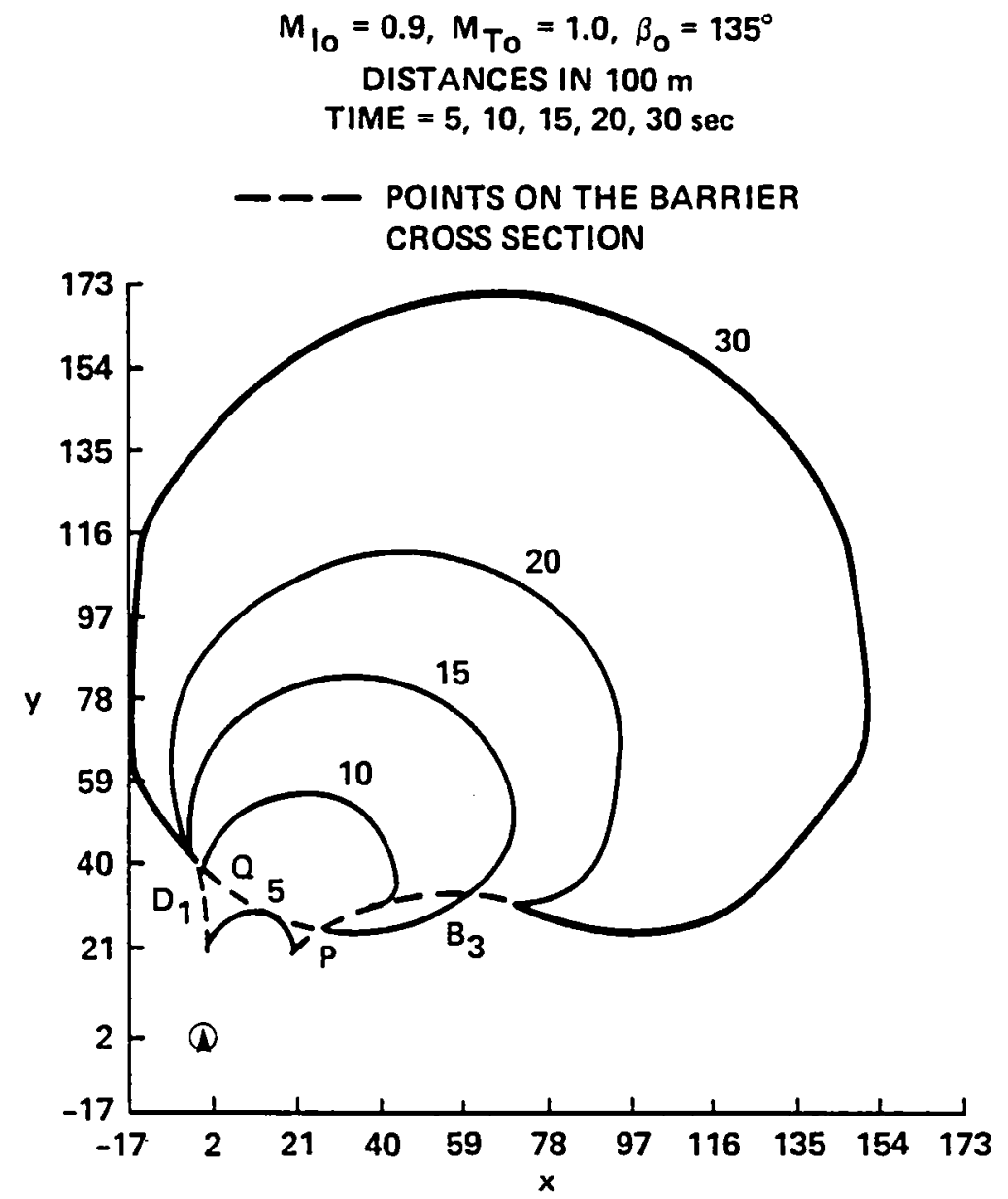

Fig. $4 b$ 


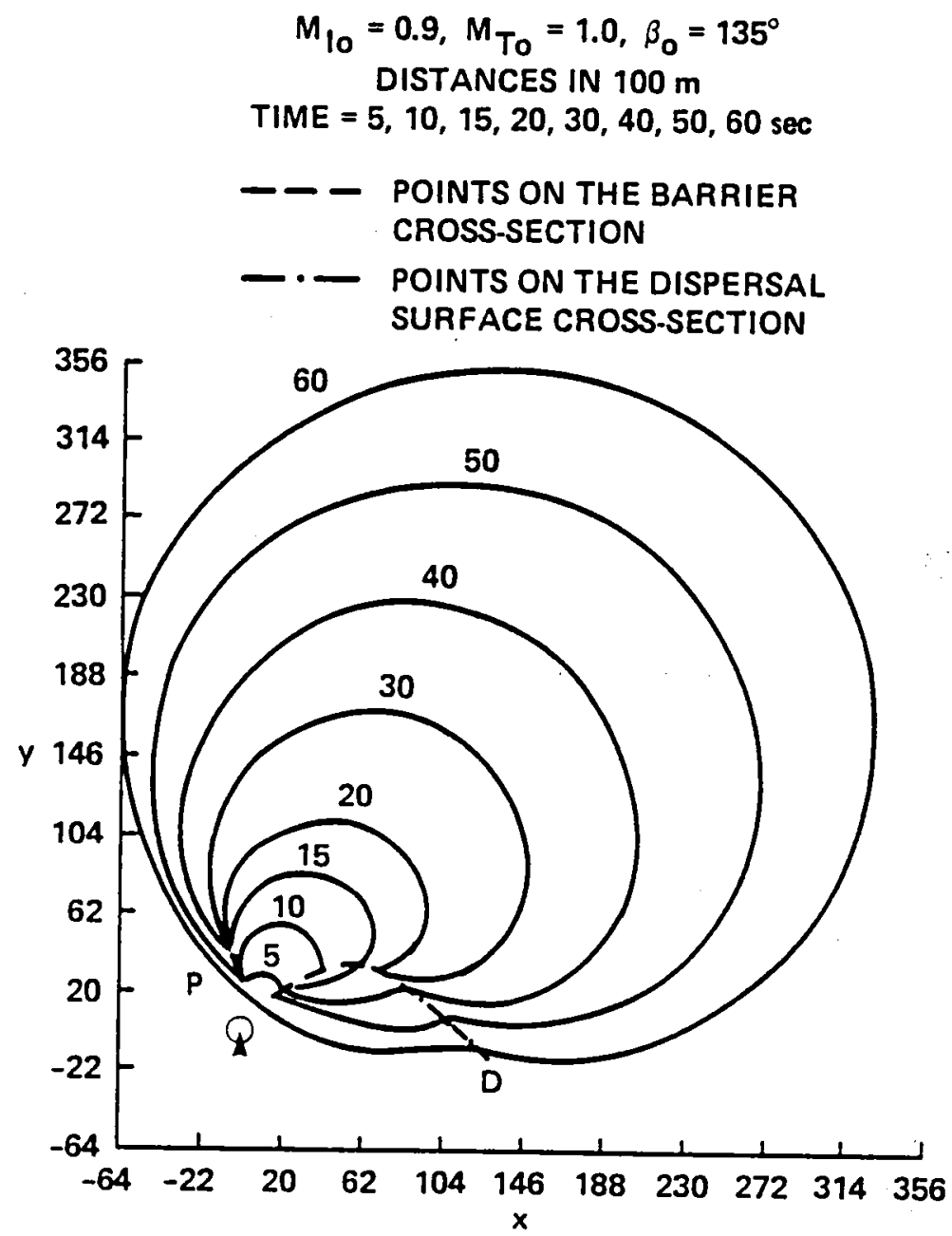

Fig. 4c 
SHOWN IN REAL SPACE

POINTS MARKED AT 10 sec INTERVALS

$M_{10}=0.9, M_{\text {To }}=1.0, \beta_{0}=135^{\circ}$

口 PATH OF INTERCEPTOR

O PATH OF TARGET

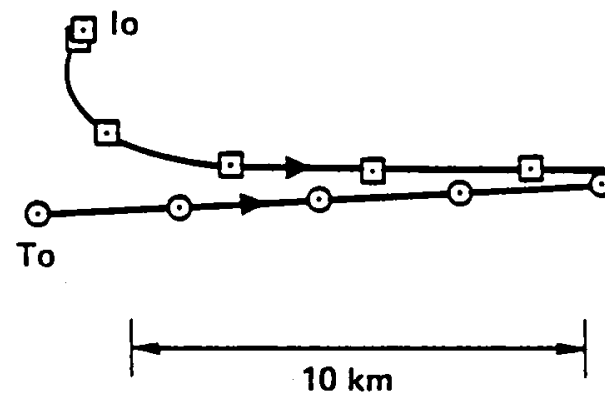

Fig. 5 
SHOWN IN REAL SPACE

POINTS MARKED AT $10 \mathrm{seC}$ INTERVALS

口 INTERCEPTOR PATH $-\beta_{f}=-4.1$ e $-4^{\circ} \quad M_{f}=1.209$

O TARGET PATH $-\beta_{o}=135^{\circ} \quad \tau_{f}=60 \mathrm{sec}$

$\triangle$ INTERCEPTOR PATH $-\beta_{f}=4.3 e-4^{\circ} \quad M_{f}=1.21$

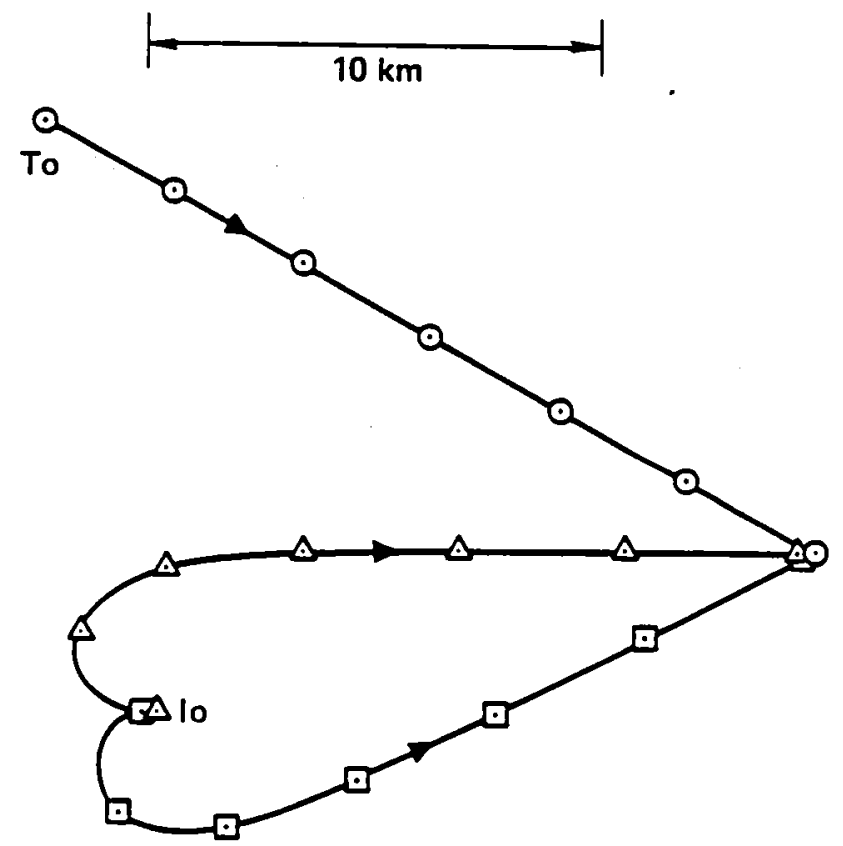

Fig. 6 


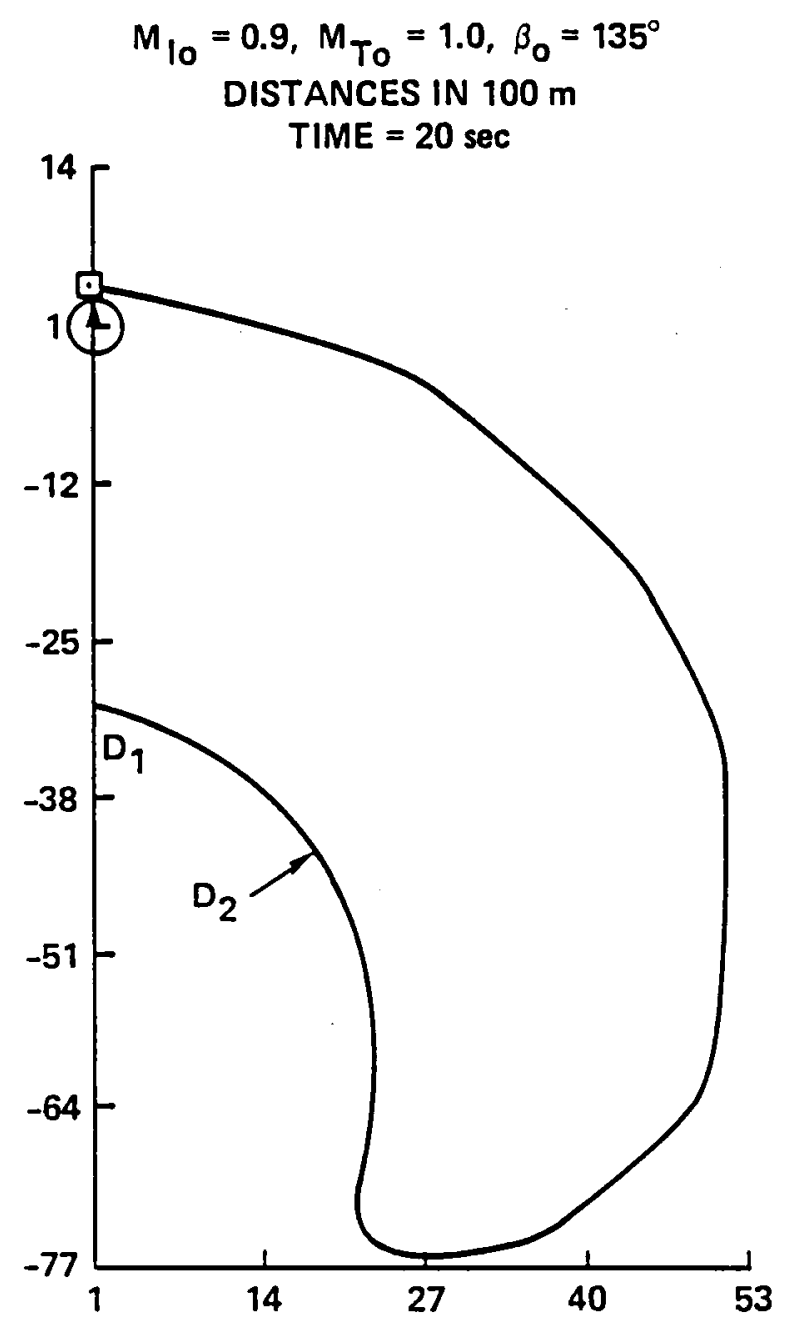

Fig. 7 
SHOWN IN REAL SPACE

POINTS MARKED AT 1 seC INTERVALS

$\square$ INTERCEPTOR PATH $-\beta_{f}=116^{\circ} \quad M_{f}=0.576$

O TARGET PATH $-\beta_{0}=0^{\circ} \quad \tau_{f}=20 \mathrm{sec}$

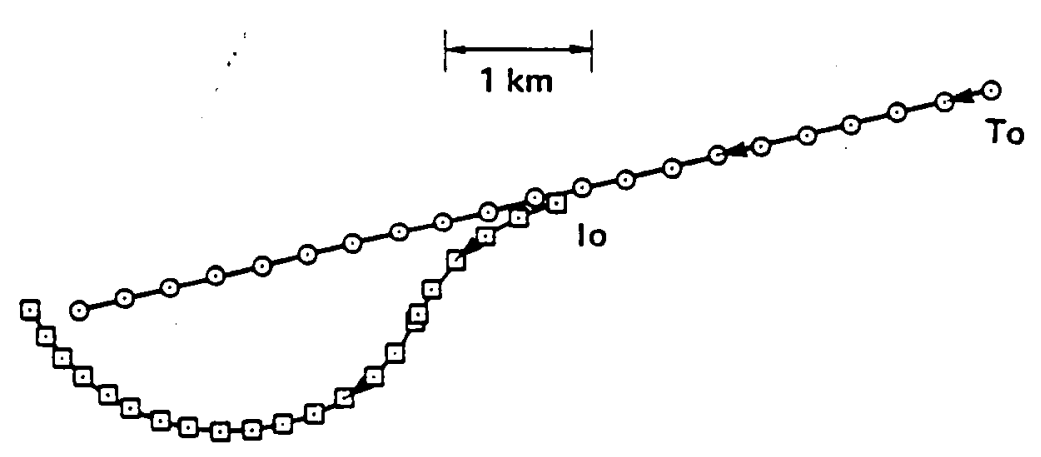

Fig. 3 
SHOWN IN REAL SPACE

POINTS MARKED AT 1 SQC INTERVALS

$\square$ INTERCEPTOR PATH $-\beta_{f}=99.5^{\circ} \quad M_{f}=0.576$
$O$ TARGET PATH $-\beta_{0}=0^{\circ} \quad \tau_{f}=20 \mathrm{sec}$
$\triangle$ INTERCEPTOR PATH $-\beta_{f}=83.5^{\circ} \quad M_{f}=0.8$

$\underset{1 \mathrm{~km}}{\longrightarrow}$

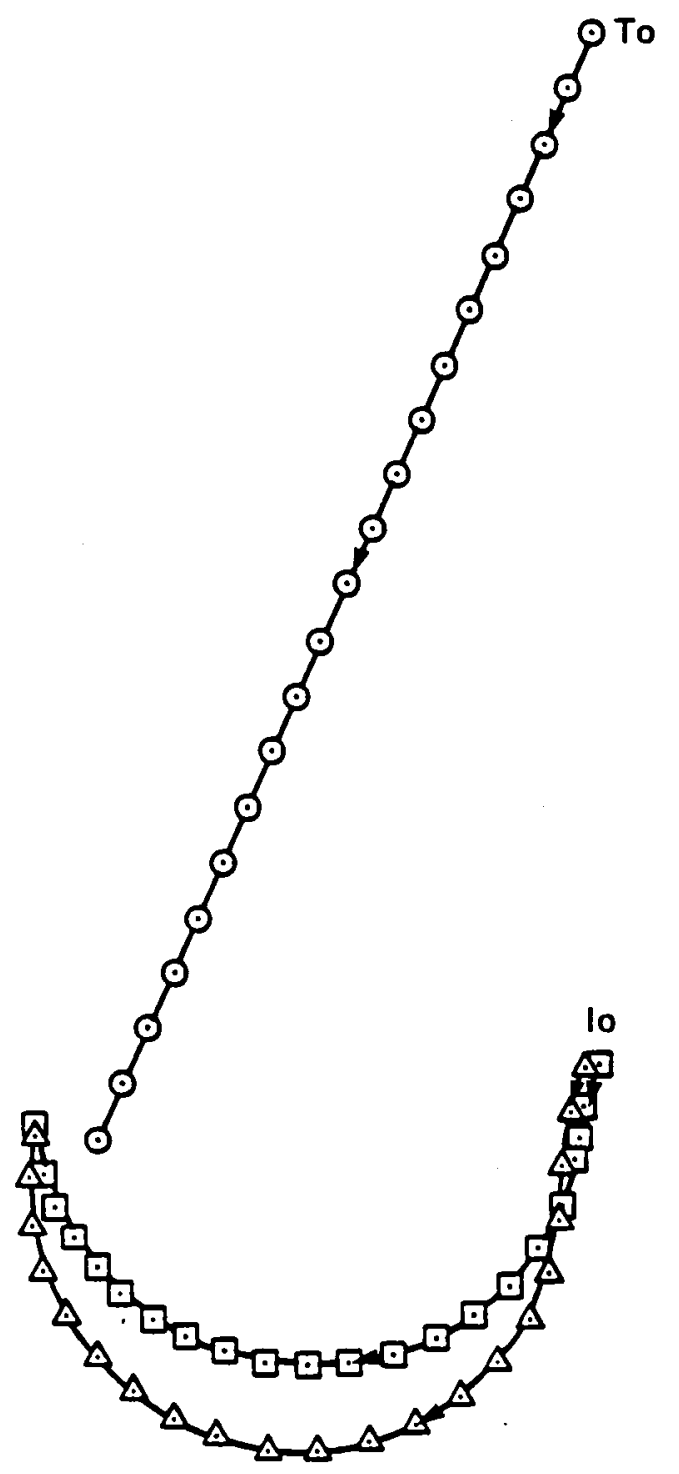

Fig. 9 


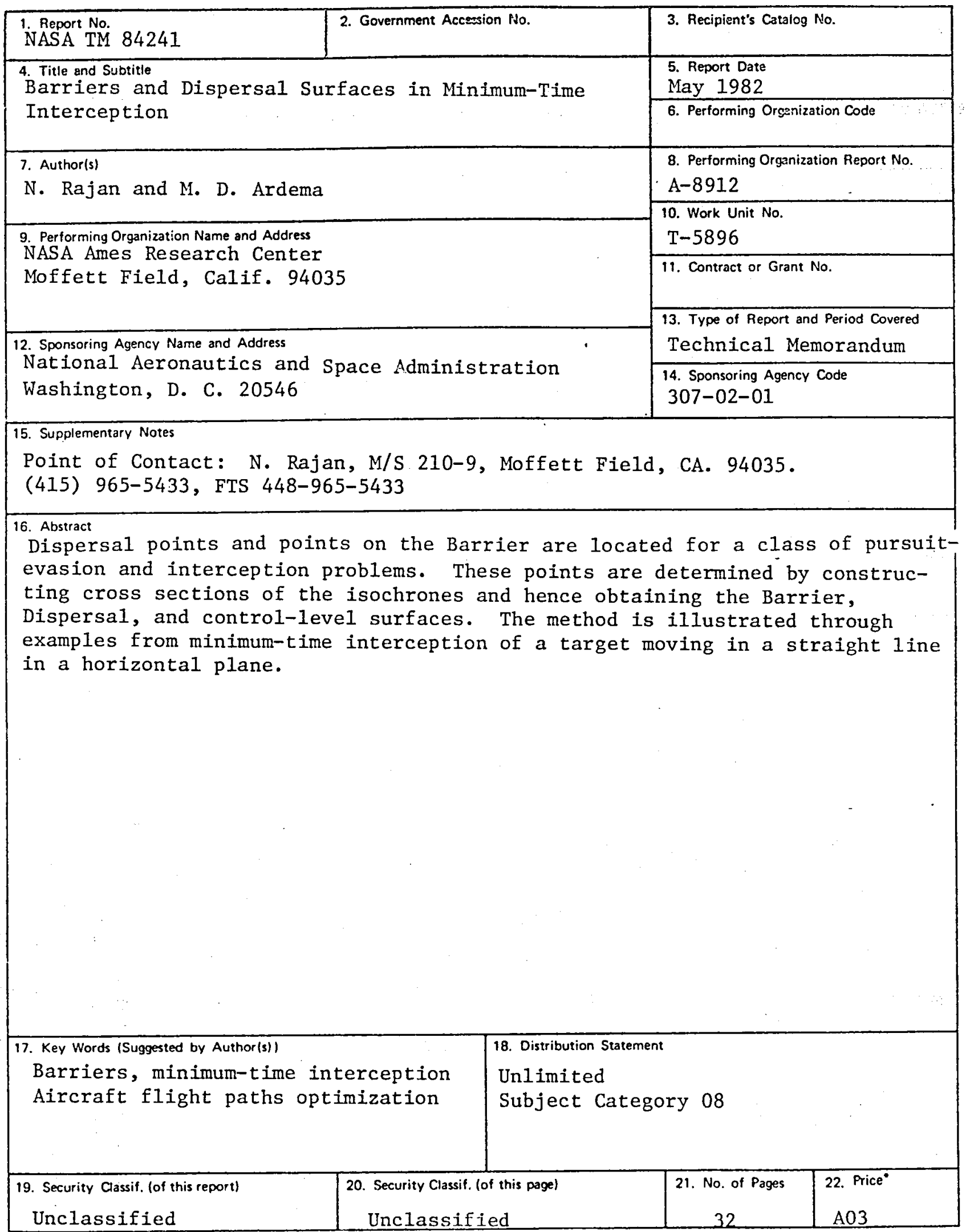

- Cor saie by the National Techniad? Information Service, Springfield, Virginia 22161 

- 
